\title{
Thermocapillary instabilities of liquid layers on an inclined plane
}

Chen-Yi Yan, Kai-Xin Hu, and Qi-Sheng Chen

Citation: Physics of Fluids 30, 082101 (2018); doi: 10.1063/1.5039149

View online: https://doi.org/10.1063/1.5039149

View Table of Contents: http://aip.scitation.org/toc/phf/30/8

Published by the American Institute of Physics

\section{Articles you may be interested in}

A new experimental method based on volume measurement for determining axial scaling during breakup of drops and liquid threads

Physics of Fluids 30, 082102 (2018); 10.1063/1.5030330

Vibrational modes prediction for water-air bubbles trapped in circular microcavities

Physics of Fluids 30, 082001 (2018); 10.1063/1.5037328

Thermocapillary instability of self-rewetting films on vertical fibers

Physics of Fluids 30, 082104 (2018); 10.1063/1.5043482

The hydrodynamic behavior of a squirmer swimming in power-law fluid

Physics of Fluids 30, 083301 (2018); 10.1063/1.5045701

Dynamics of a flexible superhydrophobic surface during a drop impact

Physics of Fluids 30, 072102 (2018); 10.1063/1.5028127

Marangoni effect on the motion of a droplet covered with insoluble surfactant in a square microchannel Physics of Fluids 30, 077101 (2018); 10.1063/1.5026874

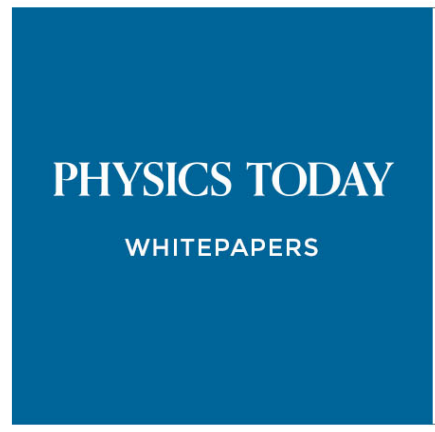

ADVANCED LIGHT CURE ADHESIVES

Take a closer look at what these environmentally friendly adhesive systems can do
READ NOW

PRESENTED BY

QMASTERBOND 


\title{
Thermocapillary instabilities of liquid layers on an inclined plane
}

\author{
Chen-Yi Yan, ${ }^{1}$ Kai-Xin Hu, ${ }^{1, a)}$ and Qi-Sheng Chen ${ }^{2,3}$ \\ ${ }^{1}$ School of Mechanical Engineering and Mechanics, Ningbo University, Ningbo, Zhejiang 315211, China \\ ${ }^{2}$ School of Engineering Science, University of Chinese Academy of Sciences, Beijing 100190, China \\ ${ }^{3}$ Key Laboratory of Microgravity, Institute of Mechanics, Chinese Academy of Sciences, Beijing 100190, China
}

(Received 7 May 2018; accepted 13 July 2018; published online 2 August 2018)

\begin{abstract}
The thermocapillary instabilities of the liquid layers on an inclined plane with zero heat flux are examined by linear stability analysis. There is an angle between the gravity and the thermocapillary force. The basic flow depends on the ratio of the gravity effect to thermocapillary effect. When the ratio is positive, the gravity always increases the velocity and destabilizes the flow. When the ratio is negative, the influence of gravity on the flow stability depends on the Prandtl $(P r)$ number. At high and moderate $P r$, the critical Marangoni number always decreases with the ratio. The vertical convection is the key to the instability mechanism at high $P r$, while the horizontal convection is dominant at moderate $P r$. By contrast, at small $P r$, the critical Marangoni number has a maximum and the energy from the basic flow is important for the perturbation energy. Published by AIP Publishing. https://doi.org/10.1063/1.5039149
\end{abstract}

\section{INTRODUCTION}

Thermocapillary flows refer to the flows caused by a temperature-induced surface tension gradient. The thermocapillary liquid layer driven by a horizontal temperature gradient arises in several different situations. In many cases, the temperature gradient on the liquid surface is used to affect the outcome, such as the liquid bridge, ${ }^{1}$ droplet migration, ${ }^{2}$ climbing films, ${ }^{3}$ and convections in closed cavities. ${ }^{4}$ It also plays a crucial role in many industrial applications, such as fusion welding, ${ }^{5}$ crystal growth stabilization, ${ }^{6}$ and heat transfer operations. $^{7}$ The thermocapillary effect on the dynamics of thin liquid films has been studied by many authors in different geometry, such as sphere, ${ }^{8}$ vertical cylinders, ${ }^{9}$ and horizontal cylinders. ${ }^{10,11}$ In the theoretical studies, the model proposed by Smith and Davis ${ }^{12}$ has been widely used, where a fluid layer above an infinite rigid plane is driven by a constant temperature gradient imposed on the interface. The oblique hydrothermal waves predicted by this model have been observed in both the experiment ${ }^{13}$ and the numerical simulation. ${ }^{14}$

The model above has also been used in the study of thermocapillary liquid layers in the presence of gravity. Garr-Peters ${ }^{4,15}$ has performed the linear stability analysis for a surface-tension-driven fluid layer subject to buoyant forces. The free surface is facing either upward or downward while the Prandtl numbers considered are between 0.01 and 10 . It was found that due to gravity, the liquid layer becomes more unstable at small Prandtl numbers. Parmentier et al. ${ }^{16}$ have examined the buoyant-thermocapillary instabilities in thin liquid layers for $0.01 \leq P r \leq 7$. Chan and $\mathrm{Chen}^{17}$ have studied the linear stability of thermocapillary fluid layers coupled with the effect of gravity for $P r=13.9$. The critical Marangoni number increases with Grashof number (a measure of gravity) while the preferred mode changes from the

a)Email: hukaixin@nbu.edu.cn oblique wave to the transverse wave. Their numerical results compare favorably with the experiment by Riley and Neitzel. ${ }^{13}$ Recently, the study of thermocapillary flows with gravity has been generalized to non-Newtonian fluids, such as the shearthinning fluid, ${ }^{18}$ the Bingham fluid, ${ }^{19}$ and the viscoelastic fluid. $^{20,21}$

It should be noted that the model of thermocapillary liquid layers used above is very different from the following two models. The first is the Benard-Marangoni convection. ${ }^{22}$ Although the gravity and thermocapillary forces exist in both the Benard-Marangoni convection and the thermocapillary liquid layer, the liquid is heated from below for the former, while for the latter, the bottom has zero heat flux. The second is the thermocapillary convection with a deformable liquid surface. ${ }^{23}$ However, the model of thermocapillary liquid layers assumes that the surface tension is big enough so that the surface deformation is negligible. ${ }^{12}$

The studies mentioned above have shown the effect of gravity on the thermocapillary convection on a horizontal plane. However, in many practical situations, the plane below the liquid is not always horizontal. Goussis and Kelly ${ }^{24}$ have examined the liquid film flowing down a heated plane. They found that there can be two kinds of instabilities, which are the surface wave and thermocapillary instabilities. The former is an interfacial instability, which is associated with the deformation of the surface. The surface wave instability in the liquid flow down an inclined plane has been investigated by Benjamin $^{25}$ and Yih. ${ }^{26}$ However, the condition for this instability may not be satisfied in the low-gravity condition, which will be explained later. The surface wave instability in the flow caused by thermocapillary shear stresses has been studied by Smith and Davis. ${ }^{27}$ They suggested that the surface wave is not preferred when the surface-tension number is large enough.

To the best of our knowledge, no comprehensive study has been carried out for the thermocapillary instabilities in the liquid layer on an inclined plane with zero heat flux. Thus, in 
the present work, the study of thermocapillary liquid layers has extended from the flow on a horizontal plane to that on an inclined plane. The surface wave is not considered. The fluid is assumed to be Newtonian and the results are presented for Prandtl numbers $0.01,1$, and 100 .

The paper is organized as follows. In Sec. II, the physical model and mathematical formulations are presented. The solution of the basic flow and the dimensionless governing equations are derived. Then in Sec. III, numerical results are obtained. The perturbation flow field is displayed and the energy mechanism is studied. After that, the instability mechanism is discussed in Sec. IV. Finally, the conclusions are drawn in Sec. V.

\section{PROBLEM FORMULATION}

An infinite liquid layer on an inclined plane is displayed in Fig. 1. The basic flow is assumed to be parallel and $u_{0}$ is the velocity. A temperature gradient is imposed on the liquid surface and the bottom has zero heat flux. For simplicity, we suppose that the surface tension is big enough such that the deformation of free surface is not considered. ${ }^{12} x, y, z$ are the streamwise, spanwise, and wall-normal direction, respectively. The liquid is in contact with an inviscid atmosphere, and the gravity is imposed in the vertical direction.

\section{A. Governing equations}

In this work, we assume that the surface tension $\sigma^{\prime}$ is related to the temperature $T$ as $\sigma^{\prime}=\sigma^{\prime}{ }_{0}-\gamma\left(T-T_{0}\right)$, where $\gamma$ is the negative rate of change of surface tension with temperature. $R$ is the Reynolds number defined as $R=\rho U d / \mu$, where $\rho, d$, and $\mu$, are the fluid density, the depth of the layer, and dynamic viscosity, respectively. $U$ is the characteristic velocity with the expression $U=\lambda \gamma d / \mu$, where $\lambda$ is the surface temperature gradient. The variation of surface tension with temperature leads to the thermocapillary effect and can be measured by the Marangoni number, which is defined as $M a=R \cdot P r$. Here $P r$ is the Prandtl number defined as $\operatorname{Pr}=\mu /(\rho \chi), \chi$ is the thermal diffusivity. The gravity effect can be measured by $G=\rho g d^{2} / \mu U$, which stands for the ratio of gravity to thermocapillary force. $g$ is the gravitational acceleration. $S=\rho d \sigma^{\prime}{ }_{0} / \mu^{2}$ is the non-dimensional surface-tension number.

The condition for the surface wave instability in the liquid flow down an inclined plane is ${ }^{25}$

$$
\frac{8}{5}>\frac{\alpha^{2} \sigma^{\prime}}{\rho d U_{0}^{2}}+\frac{d g|\sin \varphi|}{U_{0}^{2}},
$$

where $U_{0}=d^{2} g|\cos \varphi| /(2 v)$ is the velocity scale defined by gravity and $\alpha$ is the wave number in the streamwise direction. Here $v$ is the kinematic viscosity. The minimum of $\alpha$ has the scale $L^{-1}$, where is $L$ the length of the film. When $\sigma^{\prime}$ is large enough and $g$ is very small (our primary interest is the flow instability occurring in the microgravity environment), (2.1) cannot be satisfied. For example, the $1 \mathrm{cSt}$ silicone oil has $\rho=0.818 \times 10^{3} \mathrm{~kg} / \mathrm{m}^{3}, v=1 \times 10^{-6} \mathrm{~m}^{2} / \mathrm{s}$. If we set $d=1 \mathrm{~mm}, g=10^{-2} \mathrm{~kg} / \mathrm{m}^{3}, L=3.14 \mathrm{~m}, \varphi=135^{\circ}$, then $\alpha=1 / \mathrm{m}$, $\frac{\alpha^{2} \sigma^{\prime}}{\rho d U_{0}^{2}}+\frac{d g|\sin \varphi|}{U_{0}^{2}}=2044.58$. However, when $\lambda=0.1 \mathrm{~K} / \mathrm{mm}$, then $M a=78.88, G a=-0.77$, which are in the range we considered in this paper.

Moreover, the surface wave instability caused by thermocapillary shear stresses is not preferred when the surfacetension number $S$ is large enough. ${ }^{27}$ Therefore, in this paper, we only consider the thermocapillary instability in the flow with a non-deformable surface.

The magnitude of the surface deformation can be measured by the capillary number ${ }^{12}$

$$
C a=\frac{M a}{\operatorname{Pr} \cdot S} .
$$

In addition, the gravity effect on surface deformation can be measured by $\mathrm{Ca} \cdot \mathrm{G}$. In the experiments in Ref. $13, S$ and $\mathrm{Ca}$ are typically of the order of 25000 and 0.001 for the silicone oil, respectively. In Ref. 12 , it is shown that $S=2.32 \times 10^{6}$ and $C a=1.2 \times 10^{-4}$ for liquid silicon. In this work, $|G|<10$. Therefore, $\mathrm{Ca}$ and $\mathrm{Ca} \cdot \mathrm{G}$ are far less than 1, indicating that the assumption of a flat free surface is reasonable.

The gravity effects in the $x$ and $z$ directions are measured by $G a$ and $G b$, respectively,

$$
G a=G \cos \varphi, G b=G \sin \varphi .
$$

The dimensionless governing equations are given below, which are the continuity equation, the momentum equation, and the energy equation, respectively,

$$
\begin{gathered}
\nabla \cdot \mathbf{u}=0 \\
R\left(\frac{\partial \mathbf{u}}{\partial t}+\mathbf{u} \cdot \nabla \mathbf{u}\right)=-\nabla p+\nabla \cdot \boldsymbol{\tau}+G a \mathbf{e}_{x}-G b \mathbf{e}_{z} \\
\frac{\partial T}{\partial t}+\mathbf{u} \cdot \nabla T=\frac{1}{M a} \nabla^{2} T .
\end{gathered}
$$

Here $\mathbf{u}, p$, and $T$ stand for the velocity field, pressure, and temperature, respectively. $\boldsymbol{\tau}$ is the stress. For a Newtonian fluid,

$$
\boldsymbol{\tau}=2 \cdot \mathbf{S},
$$
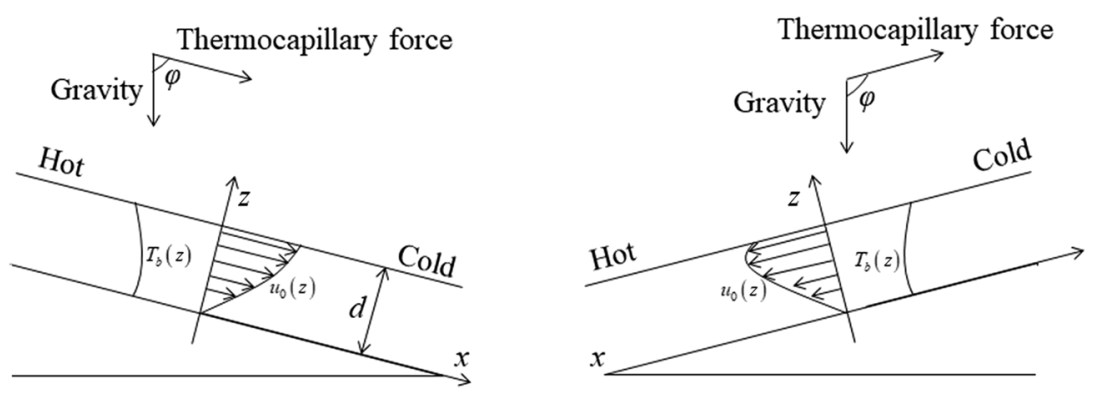

FIG. 1. The thermocapillary liquid layer on a rigid inclined plane. 
where $\mathbf{S}$ is the strain-rate tensor with the form $\mathbf{S}=\nabla \mathbf{u}+(\nabla \mathbf{u})^{T}$. It should be noted that (2.5) is a little different from the momentum equation in the buoyant-thermocapillary convection on a horizontal plane. ${ }^{4,15}$ For the latter, the variation of density with temperature leads to the buoyancy effect, which can be measured by the Bond number,

$$
B o=\frac{\rho g \kappa d^{2}}{\gamma} .
$$

Here $\kappa$ is the thermal expansion coefficient. However, in the present work, the ratio of buoyancy to gravity is

$$
B o / G=\kappa \lambda d .
$$

For the silicone oil, $\kappa, \gamma, \rho$, and $\mu$ are of the order of $10^{-3} \mathrm{~K}$, $10^{-4} \mathrm{~N} /(\mathrm{K} \cdot \mathrm{m}), 10^{3} \mathrm{~kg} / \mathrm{m}^{3}$, and $10^{-3} \mathrm{~Pa} \cdot \mathrm{s}$, respectively. When $\lambda$ and $d$ are of the order of $0.01 \mathrm{~K} / \mathrm{mm}$ and $1 \mathrm{~mm}$, respectively, then $B o / G \sim O\left(10^{-5}\right), R=M a / P r \sim O(1)$; when $\lambda$ and $d$ are of the order of $1 \mathrm{~K} / \mathrm{mm}$ and $10 \mathrm{~mm}$, respectively, then $B o / G \sim$ $O\left(10^{-2}\right), R=M a / P r \sim O\left(10^{4}\right)$. Furthermore, it has been found that the buoyancy mode causes instability only when the angle of inclination is small, ${ }^{28}$ which is not our primary interest here. Therefore, we neglect the effect of buoyancy in this paper for simplicity.

The boundary conditions are set as follows. In (2.10), there are no slip and zero heat flux on the plane. On the free surface, the first two equations in (2.11) stand for the relation between the temperature gradient and shear stress caused by thermocapillary effects, while the third equation stands for zero heat flux. $\tau_{i j}$ is the component of the stress tensor $\boldsymbol{\tau}$, and the subscripts 1,2 , and 3 in $\tau_{i j}$ stand for the $x, y$, and $z$ directions, respectively,

$$
\begin{gathered}
\left.u\right|_{z=0}=0,\left.\frac{\partial T}{\partial z}\right|_{z=0}=0, \\
\left.\tau_{13}\right|_{z=1}=-\left.\frac{\partial T}{\partial x}\right|_{z=1},\left.\tau_{23}\right|_{z=1}=-\left.\frac{\partial T}{\partial y}\right|_{z=1},\left.\frac{\partial T}{\partial z}\right|_{z=1}=0 .
\end{gathered}
$$

We assume the basic flow is parallel while its temperature is linear in $x$ as imposed plus a distribution in $z$ as follows:

$$
\mathbf{u}=\left(u_{0}(z), 0,0\right), T(x, z)=-x+T_{b}(z) .
$$

The solution has the form

$$
\begin{gathered}
u_{0}=G a\left(-\frac{1}{2} z^{2}+z\right)+z \\
T_{b}=M a\left[G a\left(\frac{1}{24} z^{4}-\frac{1}{6} z^{3}+\frac{1}{8}\right)-\frac{1}{6}\left(z^{3}-1\right)\right] .
\end{gathered}
$$

The distributions of velocity and temperature in the basic flow are displayed in Figs. 2 and 3, respectively. It can be

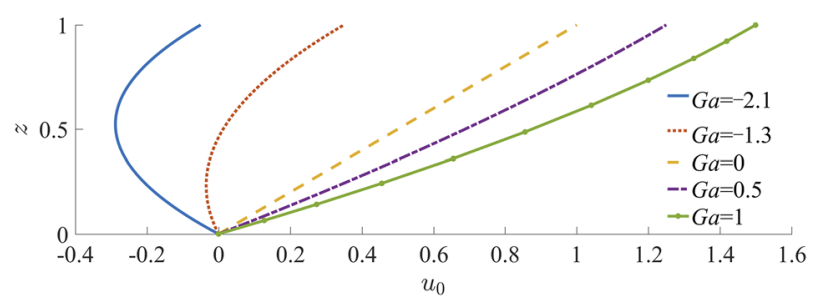

FIG. 2. The distributions of velocity for the basic flow.

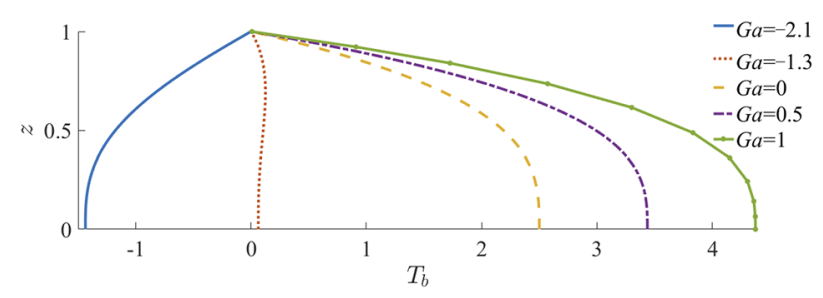

FIG. 3. The distributions of temperature at $M a=15$ for the basic flow.

seen that both the velocity and temperature increase with $G a$. When $G a>0$, the velocity is positive. The velocity profile is similar to that in the linear flow, ${ }^{12}$ and the temperature at the bottom is higher than that on the surface. When $G a=-1.3$, the velocity profile is similar to that in the return flow. ${ }^{12}$ When $G a=-2.1$, the velocity becomes negative and the temperature on the surface is higher than that at the bottom.

\section{B. Perturbation equations}

Suppose an infinitesimal perturbation in the normal mode form is added to the basic flow

$$
\begin{gathered}
(\mathbf{u}, T, P, \boldsymbol{\tau})=\left(\mathbf{u}_{0}, T_{0}, P_{0}, \boldsymbol{\tau}_{0}\right)+\boldsymbol{\delta}, \\
\boldsymbol{\delta}=(\widehat{u}, \widehat{v}, \widehat{w}, \widehat{T}, \widehat{p}, \widehat{\boldsymbol{\tau}}) \exp [\sigma t+\mathrm{i}(\alpha x+\beta y)] .
\end{gathered}
$$

Hereafter, the subscript 0 stands for the basic flow and the variables without subscript 0 stand for the perturbation. $\sigma=\sigma_{r}$ $+\mathrm{i} \sigma_{i}$, where $\sigma_{r}$ and $\sigma_{i}$ are the growth rate and frequency, respectively. The wave number and the direction of wave propagation are defined as $k=\sqrt{\alpha^{2}+\beta^{2}}$ and $\theta=\tan ^{-1}(\beta / \alpha)$, respectively. Due to symmetry, we shall confine ourselves to the case of $\theta \in\left(0^{\circ}, 180^{\circ}\right)$. This is a little different from the case in Ref. 29 where the results are performed only for $\theta \in\left(0^{\circ}, 90^{\circ}\right)$. For the latter, due to the antisymmetry of the basic flow, the wave propagation in the direction of $\theta$ is similar to the case of $180^{\circ}-\theta$. However, in this paper, the case $\theta<90^{\circ}\left(\theta>90^{\circ}\right)$ corresponds to the downstream (upstream) wave.

Introducing the (2.14) and (2.15) into the governing Eqs. (2.4)-(2.7), we can obtain the linearized perturbation equations as follows:

Continuity equation

$$
\mathrm{i} \alpha \widehat{u}+\mathrm{i} \beta \widehat{v}+D \widehat{w}=0 .
$$

Momentum equations

$$
R\left(\sigma \widehat{u}+\widehat{w} D u_{0}+u_{0} \mathrm{i} \alpha \widehat{u}\right)=-\mathrm{i} \alpha \widehat{p}+\mathrm{i} \alpha \widehat{\tau}_{11}+\mathrm{i} \beta \widehat{\tau}_{12}+D \widehat{\tau}_{13},
$$

$R\left(\sigma \widehat{v}+u_{0} \mathrm{i} \alpha \hat{v}\right)=-\mathrm{i} \beta \widehat{p}+\mathrm{i} \alpha \widehat{\tau}_{12}+\mathrm{i} \beta \widehat{\tau}_{22}+D \widehat{\tau}_{23}$,

$R\left(\sigma \widehat{w}+u_{0} \mathrm{i} \alpha \widehat{w}\right)=-\widehat{p}+\mathrm{i} \alpha \widehat{\tau}_{13}+\mathrm{i} \beta \widehat{\tau}_{23}+D \widehat{\tau}_{33}$. 
TABLE I. The critical parameters for instabilities.

\begin{tabular}{lccccc}
\hline \hline & \multicolumn{2}{c}{ Smith and Davis ${ }^{12}$} & & \multicolumn{2}{c}{ Present work } \\
\cline { 2 - 3 } \cline { 5 - 6 } Basic flow & Linear flow & Return flow & & Linear flow & Return flow \\
\hline$P r$ & 9.25 & 0.023 & & 9.25 & 0.023 \\
$M a$ & 16.7 & 9.6 & & 16.66 & 9.73 \\
Wave number & 1.48 & 0.36 & & 1.47 & 0.36 \\
Propagation angle & 90 & 76 & & 90 & 75.5 \\
Wave speed & 0 & 0.073 & & 0 & 0.072 \\
\hline \hline
\end{tabular}

Energy equation

$M a\left(\widehat{u} \frac{\partial T_{0}}{\partial x}+\widehat{w} \frac{\partial T_{0}}{\partial z}+u_{0} \mathrm{i} \alpha \widehat{T}\right)+\left(\alpha^{2}+\beta^{2}\right) \widehat{T}-D^{2} \widehat{T}=-\sigma M a \widehat{T}$.

Constitutive equations

$$
\begin{gathered}
\widehat{\tau}_{11}-(2 \mathrm{i} \alpha \widehat{u})=0, \quad \widehat{\tau}_{12}-(\mathrm{i} \alpha \widehat{v}+\mathrm{i} \beta \widehat{u})=0, \\
\widehat{\tau}_{13}-(D \widehat{u}+\mathrm{i} \alpha \widehat{w})=0, \\
\widehat{\tau}_{22}-(2 \mathrm{i} \beta \widehat{v})=0, \quad \widehat{\tau}_{23}-(\mathrm{i} \beta \widehat{w}+D \widehat{v})=0, \\
\widehat{\tau}_{33}-(2 D \widehat{w})=0 .
\end{gathered}
$$

In the momentum equations, $\widehat{p}$ can be eliminated and the following equations are derived:

$$
\begin{aligned}
& R\left[\beta\left(\widehat{w} D u_{0}+u_{0} \mathrm{i} \alpha \widehat{u}\right)-\alpha\left(u_{0} \mathrm{i} \alpha \widehat{v}\right)\right] \\
& -\beta\left(\mathrm{i} \alpha \widehat{\tau}_{11}+\mathrm{i} \beta \widehat{\tau}_{12}+D \widehat{\tau}_{13}\right) \\
& +\alpha\left(\mathrm{i} \alpha \widehat{\tau}_{12}+\mathrm{i} \beta \widehat{\tau}_{22}+D \widehat{\tau}_{23}\right)=-\sigma R(\beta \widehat{u}-\alpha \widehat{v}), \\
& R \alpha\left(D \widehat{w} \cdot D u_{0}+\widehat{w} D^{2} u_{0}+D u_{0} \cdot \mathrm{i} \alpha \widehat{u}+u_{0} \mathrm{i} \alpha D \widehat{u}\right) \\
& +R \beta\left(D u_{0} \cdot \mathrm{i} \alpha \widehat{v}+u_{0} \mathrm{i} \alpha D \widehat{v}\right)-R \mathrm{i} k^{2}\left(u_{0} \mathrm{i} \alpha \widehat{w}\right) \\
& -\left(\mathrm{i} \alpha^{2} D \widehat{\tau}_{11}+2 \mathrm{i} \alpha \beta D \widehat{\tau}_{12}+\alpha D^{2} \widehat{\tau}_{13}+\mathrm{i} \beta^{2} D \widehat{\tau}_{22}\right. \\
& \left.+\beta D^{2} \widehat{\tau}_{23}\right)+\mathrm{i} k^{2}\left(\mathrm{i} \alpha \widehat{\tau}_{13}+\mathrm{i} \beta \widehat{\tau}_{23}+D \widehat{\tau}_{33}\right) \\
& =-\sigma R\left(\alpha D \widehat{u}+\beta D \widehat{v}-\mathrm{i} k^{2} \widehat{w}\right) .
\end{aligned}
$$

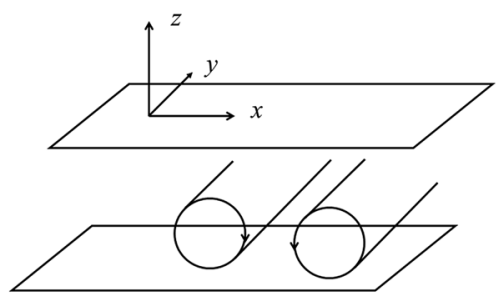

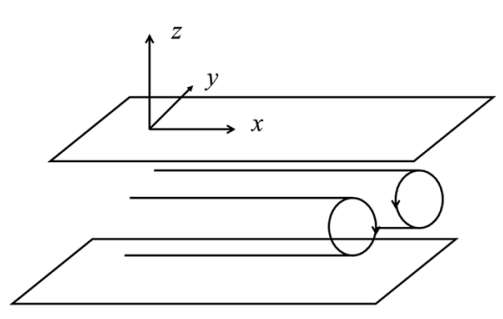

(b) (a)

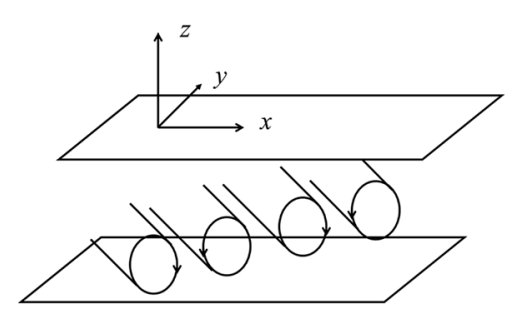

(c)
The boundary conditions for the perturbation are determined as follows:

$$
\begin{gathered}
\widehat{u}=\widehat{v}=\widehat{w}=\frac{\partial \widehat{T}}{\partial z}=0, z=0, \\
\widehat{\tau}_{13}+\mathrm{i} \alpha \widehat{T}=0, \widehat{\tau}_{23}+\mathrm{i} \beta \widehat{T}=0, \widehat{w}=0, \frac{\partial \widehat{T}}{\partial z}=0, z=1 .
\end{gathered}
$$

Then, the Chebyshev collocation method ${ }^{30}$ is used to obtain the eigenvalue $\sigma . N_{c}$ Chebyshev-collocation points $z=\left(1-\cos \left(\frac{j \pi}{N_{c}+1}\right)\right) / 2, j=1 \sim N_{c}$ are set in the flow region for Eqs. (2.16)-(2.22), while 2 points $z=0,1$ are set at the boundaries for Eqs. (2.25) and (2.26). The perturbation quantities are expanded in Chebyshev polynomials as

$$
\widehat{u}=\sum_{j=1}^{N_{c}+1} a_{j} A_{j-1}(\widehat{z})
$$

Here $\widehat{z}=1-2 z, A_{j-1}(\widehat{z})=\cos \left[(j-1) \cos ^{-1}(\widehat{z})\right]$ is the $(j-1)$-th Chebyshev polynomial and $a_{j}$ is the coefficient. Thus, the general eigenvalue problem can be solved with the form of $\mathbf{W v}=\sigma \mathbf{Z v}$, where $\mathbf{W}, \mathbf{Z}$ are two matrices and $\mathbf{v}$ is the eigenvector. The eigenvalues are obtained by using the QZ algorithm available in the Matlab-software package. In our work, the results are sufficiently accurate when $N_{c}=70 \sim 90$. In order to validate our code, we solve the same problem in previous work in Ref. 12 by setting $G a=0$ (linear flow) and $G a=-1.5$ (return flow). The critical parameters are displayed in Table I, which shows that our results are almost the same as those in Ref. 12.

\section{NUMERICAL RESULTS}

In the following, we will compute the Marangoni number $M a_{N}$ for the neutral mode $\left(\sigma_{r}=0\right)$ and find the critical Marangoni number $M a_{c}$, which is defined as follows,

$$
M a_{c}=\min _{\alpha, \beta} M a_{N}(G a, P r)
$$

There are three kinds of preferred modes (see Fig. 4), which are the streamwise wave $\left(\theta=0^{\circ}\right.$ or $\left.\theta=180^{\circ}\right)$, the spanwise stationary mode $\left(\theta=90^{\circ}, \sigma_{i}=0\right)$, and the oblique wave
FIG. 4. The schematic of different modes: (a) streamwise wave; (b) spanwise stationary mode; (c) oblique wave. 
$\left(\theta \neq 0^{\circ}, 90^{\circ}\right)$. The counter-rotating rolls are arranged periodically for all of them.

The results for $\operatorname{Pr}=100, \operatorname{Pr}=1$, and $\operatorname{Pr}=0.01$ are presented in Secs. III A, III B, and III C, respectively. The perturbation field is displayed in Sec. III D and the energy mechanism is analyzed in Sec. III E.

\section{A. $\operatorname{Pr}=100$}

The variation of $M a_{c}$ with $G a$ at $P r=100$ is displayed in Fig. 5. When $G a$ decreases, $M a_{c}$ increases obviously, especially when $G a<-1.57$. The preferred modes are the spanwise stationary mode $\left[\theta=90^{\circ}, \sigma_{i}=0\right.$, curve (e) $]$, the oblique wave $\left[\theta \neq 0^{\circ}, 90^{\circ}\right.$, curves (c), (d)], the upstream streamwise wave $\left[\theta=180^{\circ}\right.$, curves (b)], and the downstream streamwise wave $\left[\theta=0^{\circ}\right.$, curve (a) $]$.

The wave number, the propagation angle, and the wave speed corresponding to the modes in Fig. 5 are displayed in Fig. 6. It can be seen that the propagation angle of the preferred mode changes frequently when $G a<-1.32$. The wave number generally increases with $G a$ when $G a<-1.37$, but always decreases with the increase of $G a$ when $G a<-0.5$. Finally, the wave number tends to a constant $(k=0.7)$ when $G a>0$. The wave speed always decreases with $G a$ for each wave and becomes zero for the spanwise stationary mode [curve (e) in Fig. 5].

\section{B. $\operatorname{Pr}=1$}

The neutral curves at $\operatorname{Pr}=1$ are displayed in Fig. 7. $M a_{c}$ also decreases with the increase of $G a$. It is seen that the preferred mode changes frequently when $G a \in$ $(-2.2,3)$. When $G a$ decreases, the preferred modes are the spanwise stationary mode $\left[\theta=90^{\circ}\right.$, curve (d)], the downstream streamwise wave $\left[\theta=0^{\circ}\right.$, curve (c)], the oblique wave $\left[\theta \neq 0^{\circ}, 90^{\circ}\right.$, curve (b)], and the upstream streamwise wave $\left[\theta=180^{\circ}\right.$, curve (a) $]$.

In Fig. 8, we plot the wave number, the wave propagation angle, and the wave speed corresponding to the modes in Fig. 7. When $G a>-2.11$, the wave number is order 1 and changes little with $G a$; while $G a<-2.11$, the wave number is order 10 and increases obviously with the decrease of $G a$. The propagation angle decreases with the increase of $G a$ significantly for the oblique wave [curve (b) in Fig. 7]. The variation

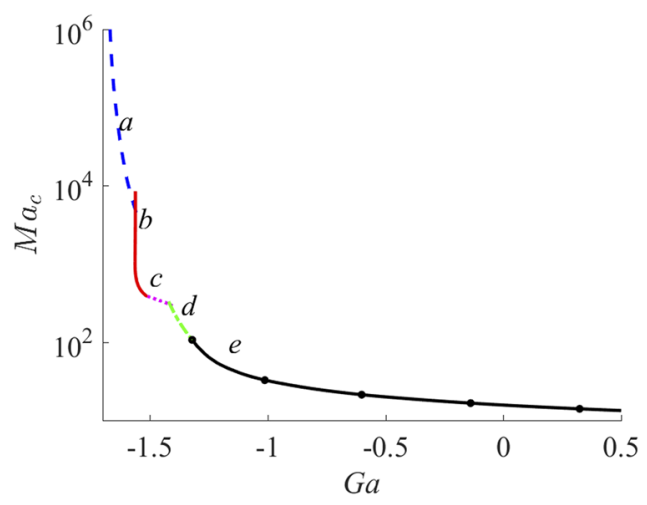

FIG. 5. The variation of $M a_{c}$ with $G a$ at $\operatorname{Pr}=100$. The curves correspond to (1) streamwise wave: (a) and (b); (2) oblique wave: (c) and (d); (3) spanwise stationary mode: (e).

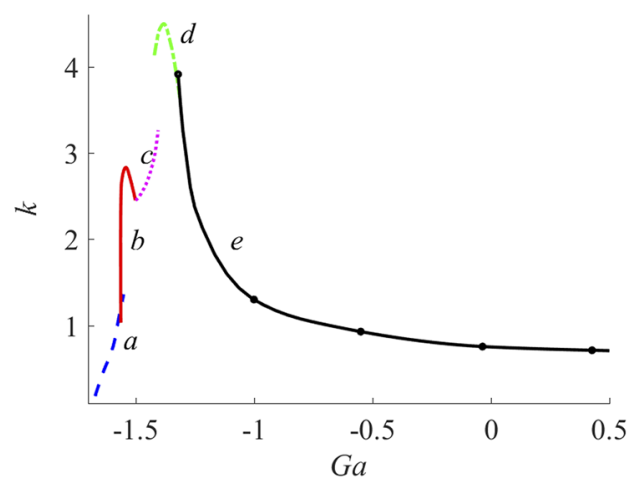

(a)

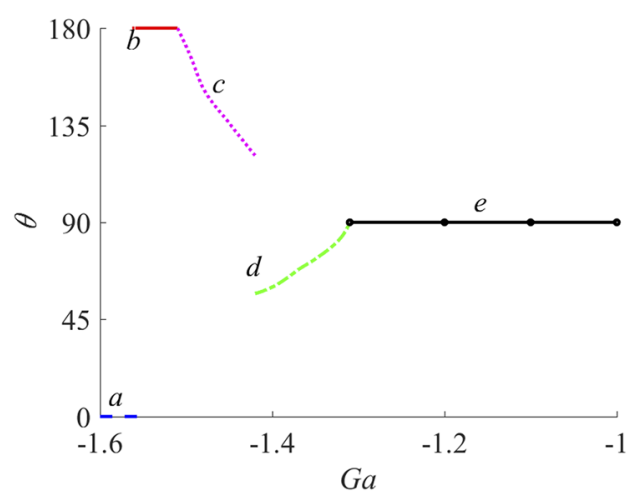

(b)

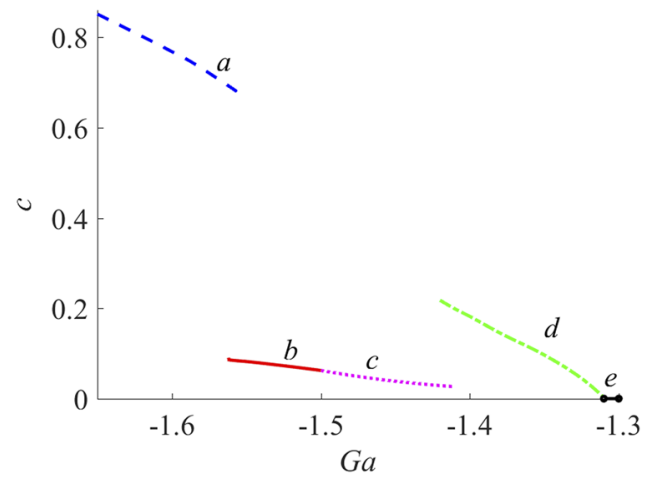

(c)

FIG. 6. The (a) wave number, (b) wave propagation angle, and (c) wave speed corresponding to the modes in Fig. 5.

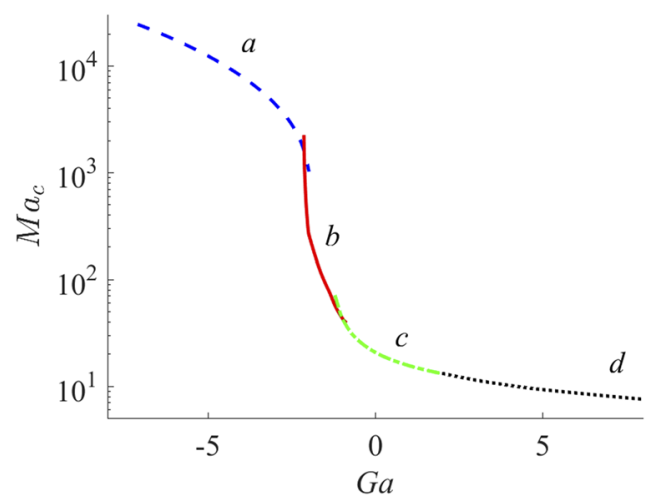

FIG. 7. The variation of $M a_{c}$ with $G a$ at $P r=1$. The curves correspond to (1) streamwise wave: (a) and (c); (2) oblique wave: (b); (3) spanwise stationary mode: (d). 


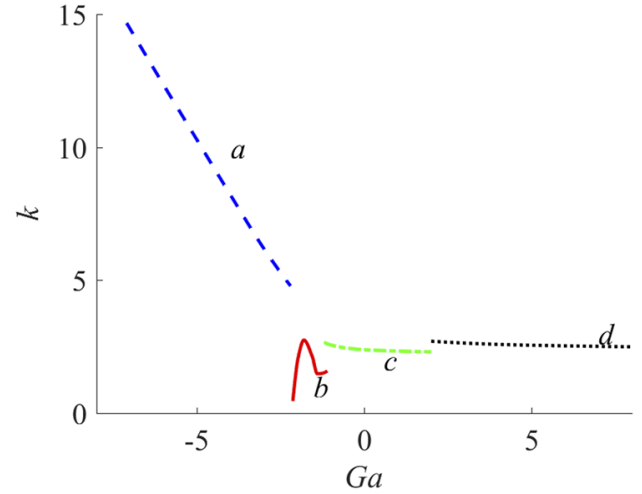

(a)

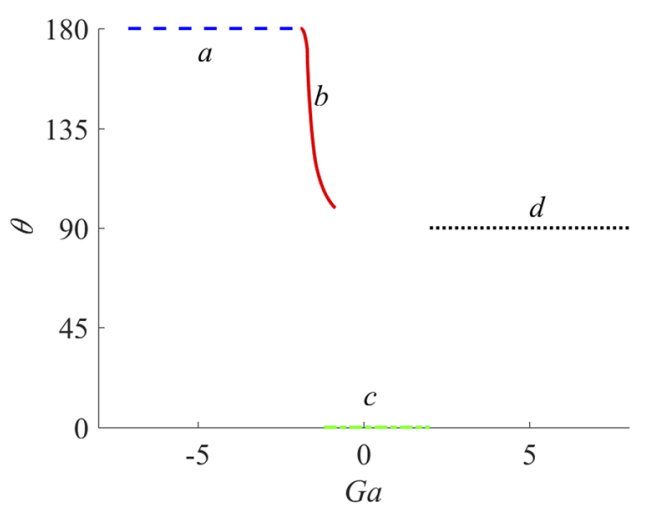

(b)

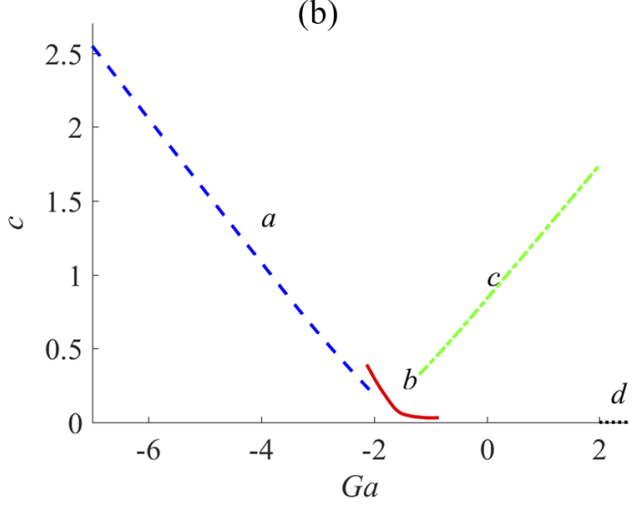

(c)

FIG. 8. The (a) wave number, (b) wave propagation angle, and (c) wave speed corresponding to the modes in Fig. 7.

of the wave speed with $G a$ is nearly linear for the streamwise waves (a) and (c).

\section{C. $P r=0.01$}

The neutral curves at $\operatorname{Pr}=0.01$ are displayed in Fig. 9 . There are two differences between the case at $P r=0.01$ and those at $\operatorname{Pr}=100$ and $\operatorname{Pr}=1$. First, the relationship between $M a_{c}$ and $G a$ is not monotonous in Fig. 9; $M a_{c}$ reaches its maximum at $G a=-2.49$. Second, the preferred mode becomes the spanwise stationary mode [curve (a) in Fig. 9] when $G a<-2.95$, while the corresponding mode is the streamwise wave [curves (a) in Figs. 5 and 7] at $\operatorname{Pr}=100$ and $\operatorname{Pr}=1$.

In Fig. 10, we plot the wave number, the wave propagation angle, and the wave speed corresponding to the modes in Fig. 9.

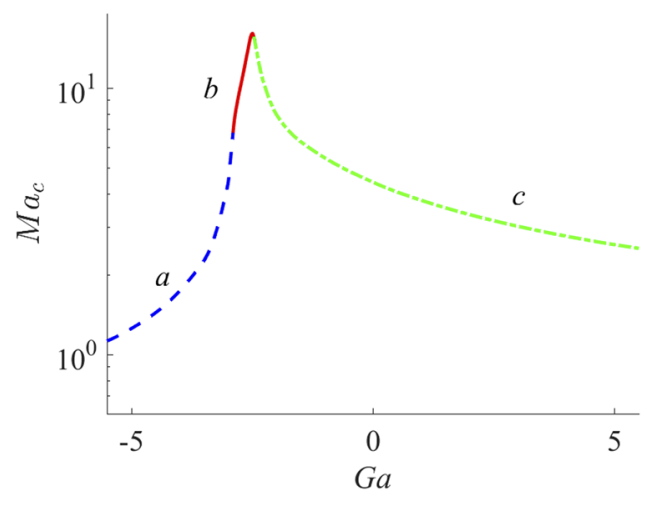

FIG. 9. The variation of $M a_{c}$ with $G a$ at $P r=0.01$. The curves correspond to (1) spanwise stationary mode: (a); (2) oblique wave: (b) and (c).

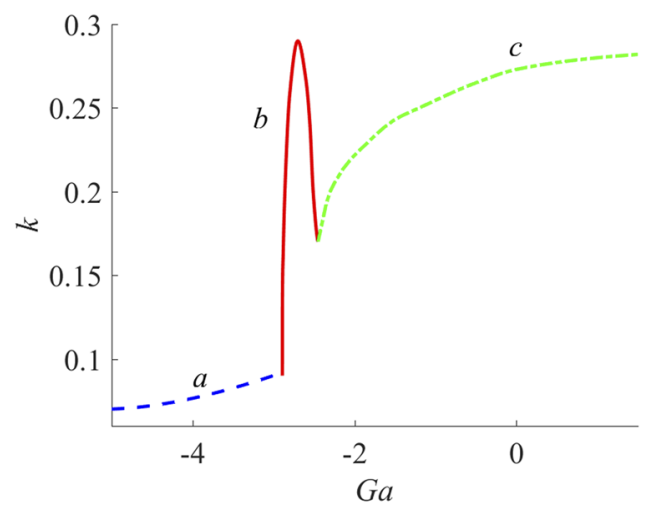

(a)

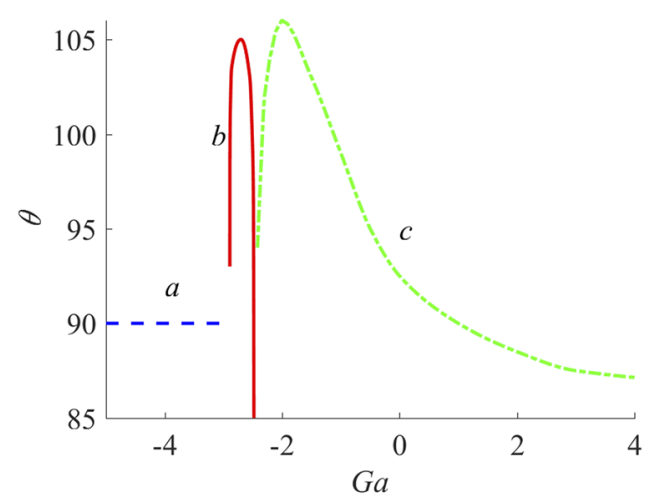

(b)

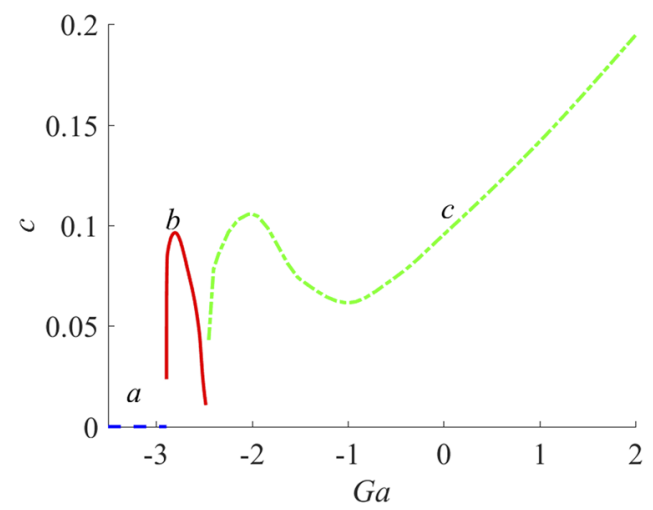

(c)

FIG. 10. The (a) wave number, (b) wave propagation angle, and (c) wave speed corresponding to the modes in Fig. 9. 
For the oblique wave (b), the wave number, the propagation angle, and the wave speed are sensitive to the change of $G a$. The wave number at $P r=0.01$ is far smaller than those at $P r=100$ and $P r=1$ (see Figs. 6 and 8). The preferred modes at $P r=0.01$ are nearly spanwise. For the oblique wave (c), the propagation angle reaches its maximum at $G a=-1.9$; the variation of its wave speed with $G a$ is nearly linear when $G a>-1$.

\section{The perturbation flow field}

The streamlines and isothermals of the preferred mode at $P r=100$ are plotted in Fig. 11. The downstream streamwise wave in Fig. 11(a) has hot spots in the interior. The streamlines near the hot spot are clockwise and almost coincide with the isothermals, which is quite different from those in the upstream waves [curves (b) and (c) in Fig. 5]. The latter are similar to the case in Fig. 12(b). For the downstream oblique wave in Fig. 11(b), there are hot spots on the surface and in the middle region of the layer $(z=0.6)$. For the spanwise stationary mode in Fig. 11(c), the isothermals are nearly vertical.

The perturbation flow field at $P r=1$ is displayed in Fig. 12. For the upstream streamwise wave in Fig. 12(a), the hot spot is near the surface $(z=0.8)$, while the temperature perturbation on the surface is very small. However, for the downstream streamwise wave, the hot spot is on the surface. For the oblique wave in Fig. 12(b), the hot spot is in the middle region of the liquid layer $(z=0.5)$.

For $P r=0.01$, the isothermals and streamlines of the preferred mode are quite similar to those of the spanwise stationary mode at $\mathrm{Pr}=100$ [Fig. 11(c)] and almost independent of $G a$. The reason can be explained as follows. The perturbation temperature mainly depends on the heat conduction at $P r=0.01$. Although the basic flow is affected by $G a$,

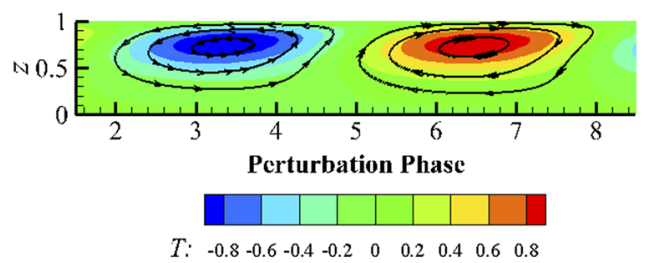

(a)

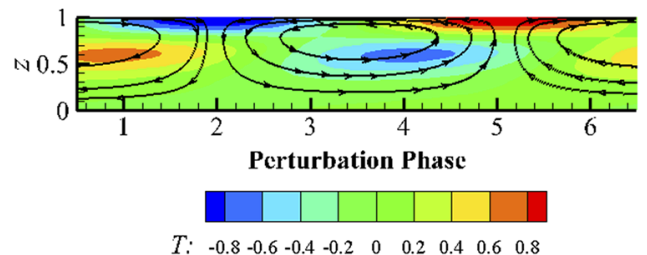

(b)

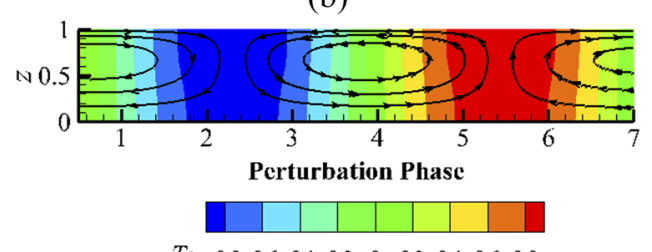

(c)

FIG. 11. The preferred mode at $\operatorname{Pr}=100$ : (a) streamwise wave $(G a=-1.59)$, (b) oblique wave $(G a=-1.38)$, and (c) spanwise stationary mode $(G a=3)$.

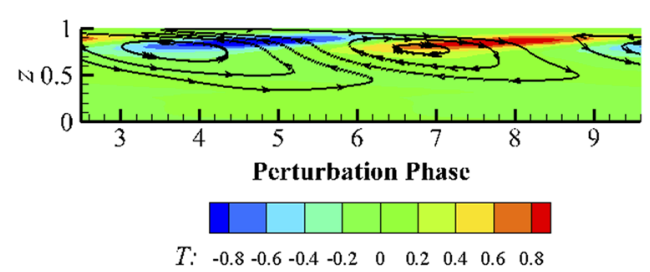

(a)

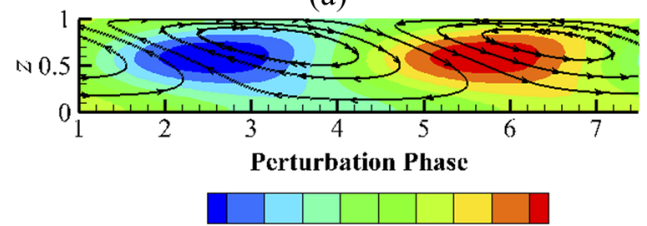

$T: \quad-0.8-0.6-0.4 \quad-0.2 \quad 0 \quad 0.2 \quad 0.4 \quad 0.6 \quad 0.8$

(b)

FIG. 12. The preferred mode at $P r=1$ : (a) streamwise wave $(G a=-3)$ and (b) oblique wave $(G a=-1.7)$.

the convective cooling of basic flow $\left(u_{0} \mathrm{i} \alpha \widehat{T}\right)$ has little influence on the perturbation temperature.

\section{E. Energy analysis}

Then we study the energy mechanism in this section. The rate of change for perturbation energy can be derived as follows: 31,32

$$
\begin{aligned}
\frac{\partial E_{k i n}}{\partial t}= & -\frac{1}{2 R} \int_{V}(\boldsymbol{\tau}: \mathbf{S}) \mathrm{d} \forall+\frac{1}{R} \oiint_{S} \mathbf{u} \cdot \boldsymbol{\tau} \cdot \mathbf{n} \mathrm{d} \mathrm{s} \\
& -\int_{V} \mathbf{u} \cdot\left((\mathbf{u} \cdot \nabla) \mathbf{u}_{0}\right) \mathrm{d} \forall=-N+M+I .
\end{aligned}
$$

Here, $N$ is the viscous dissipation, $M$ is the work done by Marangoni forces on the free surface, and $I$ is the energy from the basic flow.

The terms of perturbation energy growth at different Prandtl numbers are displayed in Table II. For $P r=100$, $I$ is almost negligible. This means that although $G a$ can change the basic flow and $M a_{c}$ significantly, there is little change for the energy mechanism. For $P r=1$, the upstream streamwise wave and the oblique wave can absorb energy from the basic flow $(I>0)$. By contrast, for the downstream streamwise wave, $I$ is negligible. For $\operatorname{Pr}=0.01, I$ is positive for both the spanwise stationary mode and the oblique wave. When $G a$ is positive, the importance of the energy from the basic flow increases with $G a$ significantly, as the velocity of the basic flow increases with $G a$. When $G a=5, I$ becomes the most important energy source for the perturbation.

TABLE II. The terms of perturbation energy growth at different Prandtl numbers. Here, SW stands for streamwise waves, OW stands for oblique waves,

\begin{tabular}{|c|c|c|c|c|c|c|c|}
\hline \multirow{2}{*}{$\begin{array}{l}P r \\
G a\end{array}$} & \multicolumn{2}{|c|}{100} & \multicolumn{3}{|c|}{1} & \multicolumn{2}{|c|}{0.01} \\
\hline & -1.59 & -1.35 & -3 & -1.6 & 0 & -5 & 5 \\
\hline Mode & SW & OW & SW & OW & SW & SP & OW \\
\hline$\frac{M}{N}$ & 1.0345 & 0.9998 & 0.7229 & 0.8232 & 1.0169 & 0.9583 & 0.4433 \\
\hline$\frac{I}{N}$ & -0.0345 & 0.0002 & 0.2771 & 0.1768 & -0.0169 & 0.0418 & 0.5571 \\
\hline
\end{tabular}
and SP stands for spanwise stationary modes. 


\section{DISCUSSION}

In this section, we will discuss the instability mechanism and the properties of preferred modes. It can be seen from the perturbation equations (2.17)-(2.20) that the gravity can influence the flow stability by changing the basic flow. The cases for three kinds of Prandtl numbers are discussed separately.

\section{A. $\operatorname{Pr}=\mathbf{1 0 0}$}

For $P r=100$, the energy analysis shows that $I$ is negligible and the thermocapillary force is the driving force for the perturbation while the perturbation stress causes damping.

For the streamwise wave [curve (a) in Fig. 5], when $G a$ decreases, the vertical gradient of temperature for the basic flow $\left(\left|\frac{\partial T_{0}}{\partial z}\right|\right)$ becomes larger. So the vertical convective cooling $\left(\left|\widehat{w} \frac{\partial T_{0}}{\partial z}\right|\right)$ increases, which decreases the temperature of the hot spot. Thus, the flow becomes more stable, and $M a_{c}$ decreases with $G a$.

For the downstream oblique wave [curve (d) in Fig. 5], the basic flow is similar to the return flow. ${ }^{12}$ There are hot spots both on the surface and in the middle region of the liquid layer [see Fig. 11(b)]. By contrast, the hot spots can only appear in the middle region of the layer for the case of without gravity. ${ }^{33}$ The reason is that the heat convec-

tion by the basic flow $\left(u_{0} i \alpha \widehat{T}\right)$ can heat the hot spot on the surface. When $G a=-1.3$, the velocity on the surface $\left(u_{0}=0.35\right)$ is larger than the case of without gravity on the surface $\left(u_{0}=0.25\right)$ for the return flow. Therefore, the heat convection becomes larger, and the hot spots also appear on the surface.

For the spanwise stationary mode [curve (e) in Fig. 5], the temperature at the bottom is higher than that on the surface for the basic flow. The instability mechanism is similar to the classical Marangoni convection. ${ }^{12}$ When $G a$ increases, the flow becomes more unstable as the vertical temperature gradient of the basic flow becomes larger.

\section{B. $\operatorname{Pr}=1$}

For the upstream streamwise wave [curve (a) in Fig. 7], $I$ is important and much larger than those of other modes at $P r=1$ (see Table II). This suggests that the velocity of the basic flow is very important for the perturbation. The computation shows that the heat convection by the basic flow $\left(u_{0} \mathrm{i} \alpha \widehat{T}\right)$ is dominant for the temperature field. As $u_{0}<0$, the disturbance propagates in the negative $x$ direction. The maximum of $\left|u_{0}\right|$ appears in the interior (see Fig. 2), so we cannot find the hot spot on the surface [Fig. 12(b)]. The wave number corresponding to the preferred mode is higher than those at $P r=100$ and $\operatorname{Pr}=0.01$. The reason is that a higher wave number leads to a larger heat convection $\left(u_{0} \mathrm{i} \alpha \widehat{T}\right)$. Therefore, the liquid layer is more unstable.

For the oblique wave [curve (b) in Fig. 7], the heat convection by the basic flow $\left(u_{0} \mathrm{i} \alpha \widehat{T}\right)$ is still dominant. Therefore, the instability mechanism is similar to the upstream streamwise wave. For the spanwise stationary mode [curve (d) in Fig. 7], the temperature at the bottom is higher than that on the surface. The instability mechanism is same as that at $\operatorname{Pr}=100$.

The downstream oblique wave is preferred for $P r=100$ and 0.01 at certain $G a$, but it is not for $P r=1$ at any $G a$. The reason can be explained as follows. The downstream oblique wave at $\operatorname{Pr}=0.01$ appears at $G a>1$, where the average velocity of the basic flow has $\bar{u}_{0}=\int_{0}^{1} u_{0}(z) \mathrm{d} z>\frac{5}{6}$. As the inertial forces are the dominant driving force in the layer, the wave is downstream. However, the convection is important for high and moderate $P r$. The hot spot of the downstream oblique wave at $P r=100$ is on the surface, where $u_{0}>0$; therefore, the wave propagates downstream. By contrast, the hot spot of the oblique wave at $P r=1$ is in the middle of the layer [see Fig. 12(b)], where $u_{0}<0$. Thus, the wave propagates upstream.

\section{C. $P r=0.01$}

For $\operatorname{Pr}=0.01, I$ is important for the perturbation energy. When $|G a|$ is large enough, both $I / N$ and the streamwise perturbation velocity $(\hat{u})$ increase with $|G a|$. As the hot spot is heated by the horizontal heat convection $\left(\hat{u} \frac{\partial T_{0}}{\partial x}\right)$, the critical Marangoni number decreases with the increase of $|G a|$.

For small $\mathrm{Pr}$, $\mathrm{Smith}^{33}$ has suggested that the key to the mechanism is the inertial-driven streamwise flow for both the linear flow and return flow. The hot spot on the surface is heated by the horizontal convection $\left(\widehat{u} \frac{\partial T_{0}}{\partial x}\right)$. Thus, $|\widehat{u}|$ at the hot spot cannot be very small. There must be an angle between the wave vector and the $x$ direction. So the streamwise waves are not preferred at $\mathrm{Pr}=0.01$.

The physical relevance of preferred modes to Prandtl number can be seen as follows. For high and moderate $P r$, the heat convection is crucial for the temperature distribution. When $G a$ is positive, the bottom is hotter than the surface. Thus, there is the possibility that a stationary convective instability can arise in this case. The preferred modes at high and moderate $P r$ are both spanwise stationary mode when $G a$ is large enough. However, when the bottom is cooler than the surface $(G a<0)$, the streamwise wave is preferred. The vertical and horizontal convections are dominant at high and moderate $\mathrm{Pr}$, respectively. At small $P r$, the heat conduction is more important, so the instability is not very sensitive to the change of temperature distribution in the basic flow. The wave propagation angles for three modes in Fig. 10(b) are close and their mechanisms are similar.

\section{CONCLUSION}

In this paper, the linear stability of thermocapillary liquid layers on an inclined plane is examined. There is an angle $\varphi$ between the gravity and the thermocapillary force. The flow depends on $G a$, which is defined as the ratio of the gravity effect in the streamwise direction to the thermocapillary effect.

When $\varphi \in\left(0^{\circ}, 90^{\circ}\right), G a>0$. Both the temperature and the velocity of the basic flow increase with $G a$. By contrast, the critical Marangoni number $M a_{c}$ decreases with the increase of $\mathrm{Ga}$. At high $\mathrm{Pr}$, the preferred mode is the spanwise stationary mode, while for small $\mathrm{Pr}$, it becomes the oblique wave. 
When $\varphi \in\left(90^{\circ}, 180^{\circ}\right), G a<0$. Both the temperature and the velocity decrease with $G a$. However, the influence of $G a$ on the flow stability depends on $P r . M a_{c}$ always decreases with $G a$ at high and moderate $P r$, but has a maximum at small $P r$. When $G a$ decreases, four kinds of preferred modes are excited at high $\mathrm{Pr}$, which are the spanwise stationary mode, the oblique wave, the upstream streamwise wave, and the downstream streamwise wave. At moderate $\mathrm{Pr}$, two kinds of streamwise waves appear, which are downstream and upstream. The wave number is large for the latter. At small $\mathrm{Pr}$, the preferred mode changes from the oblique wave to the spanwise stationary mode when $|G a|$ is large enough. There are many kinds of perturbation flow fields at high and moderate $P r$.

The instability mechanism suggests that at high $P r$, the flow stability is mainly affected by the temperature of the basic flow. When $\mathrm{Ga}$ decreases, the flow becomes more stable as the vertical convective cooling increases. By contrast, at moderate $\mathrm{Pr}$, the horizontal heat convection of the basic flow is the key to the mechanism. At small Pr, the energy from the basic flow is important for the perturbation energy. When $|G a|$ is large enough, the kinetic energy of perturbation increases with $|G a|$, which leads to the decrease of $M a_{c}$.

\section{ACKNOWLEDGMENTS}

This work has been supported by the joint fund of the satellite science research of space science of the National Natural Science Foundation of China and Chinese Academy of Sciences (No. U1738119), National Natural Science Foundation of China (Nos. 11402271 and 11532015), and sponsored by K. C. Wong Magna Fund in Ningbo University.

${ }^{1}$ K. Motegi, M. Kudo, and I. Ueno, "Linear stability of buoyant thermocapillary convection for a high-Prandtl number fluid in a laterally heated liquid bridge," Phys. Fluids 29, 044106 (2017).

${ }^{2}$ A. Corbett and S. Kumar, "Spreading of thin droplets of perfect and leaky dielectric liquids on inclined surfaces," Langmuir 32, 6606-6617 (2016).

${ }^{3}$ A. E. Hosoi and J. W. M. Bush, "Evaporative instabilities in climbing films," J. Fluid Mech. 442, 217-239 (2001).

${ }^{4}$ J. M. Garr-Peters, "The neutral stability of surface-tension driven cavity flows subject to buoyant forces-II. Oblique disturbances," Chem. Eng. Sci. 47, 1265-1276 (1992).

${ }^{5}$ T. Debroy and S. A. David, "Physical processes in fusion welding," Rev. Mod. Phys. 67, 85-112 (1995).

${ }^{6}$ L. Braescu, S. Epure, and T. Duffar, Crystal Growth Processes Based on Capillarity: Czochralski, Floating Zone, Shaping and Crucible Techniques (Wiley, 2010).

${ }^{7}$ C. Dreiser and H. Bart, "Falling film break-up and thermal performance of thin polymer film heat exchangers," Int. J. Therm. Sci 100, 138-144 (2016).

${ }^{8}$ D. Kang, A. Nadim, and M. Chugunova, "Marangoni effects on a thin liquid film coating a sphere with axial or radial thermal gradients," Phys. Fluids 29, 072106 (2017).

${ }^{9}$ Z. Ding and T. N. Wong, "Three-dimensional dynamics of thin liquid films on vertical cylinders with Marangoni effect," Phys. Fluids 29, 011701 (2017).
${ }^{10}$ T. C. Kumawat and N. Tiwari, "Hydrodynamic stability of thermoviscous liquid film inside a rotating horizontal cylinder: Heating and cooling effects," Phys. Fluids 30, 032103 (2018).

${ }^{11}$ T. C. Kumawat and N. Tiwari, "Stability analysis of the rimming flow inside a uniformly heated rotating horizontal cylinder," Phys. Fluids 29, 032102 (2017).

${ }^{12}$ M. K. Smith and S. H. Davis, "Instabilities of dynamic thermocapillary liquid layers. I. Convective instabilities,” J. Fluid Mech. 132, 145-162 (1983).

${ }^{13}$ R. J. Riley and G. P. Neitzel, "Instability of thermocapillary-buoyancy convection in shallow layers. Part 1. Characterization of steady and oscillatory instabilities," J. Fluid Mech. 359, 143-164 (2000).

${ }^{14}$ Y. R. Li, N. Imaishi, T. Azami, and T. Hibiya, "Three-dimensional oscillatory flow in a thin annular pool of silicon melt," J. Cryst. Growth 260, 28-42 (2004).

${ }^{15}$ J. M. Garr-Peters, "The neutral stability of surface-tension driven cavity flows subject to buoyant forces-I. Transverse and longitudinal disturbances," Chem. Eng. Sci. 47, 1247-1264 (1992).

${ }^{16}$ P. M. Parmentier, V. C. Regnier, and G. Lebon, "Buoyant-thermocapillary instabilities in medium-Prandtl-number fluid layers subject to a horizontal temperature gradient," Int. J. Heat Mass Transfer 36, 2417-2427 (1993).

${ }^{17}$ C. L. Chan and C. F. Chen, "Effect of gravity on the stability of thermocapillary convection in a horizontal fluid layer," J. Fluid Mech. 647, 91-103 (2010).

${ }^{18} \mathrm{~K} . \mathrm{Hu}, \mathrm{M} . \mathrm{He}, \mathrm{Q}$. Chen, and R. Liu, "Linear stability of thermocapillary liquid layers of a shear-thinning fluid," Phys. Fluids 29, 073101 (2017).

${ }^{19} \mathrm{~K} . \mathrm{Hu}, \mathrm{M} . \mathrm{He}, \mathrm{Q}$. Chen, and R. Liu, "On the stability of thermocapillary convection of a Bingham fluid in an infinite liquid layer," Int. J. Heat Mass Transfer 112, 993-1002 (2018).

${ }^{20} \mathrm{~K}$. Hu, M. He, Q. Chen, and R. Liu, "Effect of gravity on the stability of viscoelastic thermocapillary liquid layers," Int. J. Heat Mass Transfer 123, 776-786 (2018).

${ }^{21}$ M. Tong, L. Yang, and Q. Fu, "Thermocapillary instability of a twodimensional viscoelastic planar liquid sheet in surrounding gas," Phys. Fluids 26, 033105 (2014).

${ }^{22}$ Y. Li, L. Jia, L. Zhang, Y. Hu, and J. Yu, "Direct numerical simulation of Rayleigh-Benard-Marangoni convection of cold water near its density maximum in a cylindrical pool," Int. J. Therm. Sci. 124, 327-337 (2018).

${ }^{23}$ R. Buckingham, M. Shearer, and A. Bertozzi, "Thin film traveling waves and the Navier slip condition," Siam J. Appl. Math. 63, 722-744 (2003).

${ }^{24}$ D. A. Goussis and R. E. Kelly, "Surface wave and thermocapillary instabilities in a liquid film flow," Phys. Fluids 223, 25-45 (1991).

${ }^{25}$ T. B. Benjamin, "Wave formation in laminar flow down an inclined plane," J. Fluid Mech. 2, 554-573 (1957).

${ }^{26}$ C. Yih, "Stability of liquid flow down an inclined plane," Phys. Fluids 6, 321-334 (1963).

${ }^{27}$ M. K. Smith and S. H. Davis, "Instabilities of dynamic thermocapillary liquid layers. Part 2. Surface-wave instabilities," J. Fluid Mech. 132, 145162 (1983).

${ }^{28}$ R. E. Kelly and D. A. Goussis, "Instability of a liquid film flowing down a heated inclined plane," Heat Transfer 5, 319 (1982).

${ }^{29}$ A. S. Ortiz-perez and L. A. Davalos-orozco, "Convection in a horizontal fluid layer under an inclined temperature gradient," Phys. Fluids 23, 084107 (2011).

${ }^{30}$ P. J. Schmid and D. S. Henningson, Stability and Transition in Shear Flows, (Springer-verlag, 2001), Vol. 412, pp. 57-59.

${ }^{31}$ M. Wanschura, V. Shevtsova, H. Kuhlmann, and H. Rath, "Convective instability mechanisms in thermocapillary liquid bridges," Phys. Fluids 7, 912-925 (1995).

${ }^{32}$ K. Hu, J. Peng, and K. Zhu, "The linear stability of plane Poiseuille flow of burgers fluid at very low Reynolds numbers," J. Non-Newtonian Fluid Mech. 167, 87-94 (2012).

${ }^{33}$ M. K. Smith, "Instability mechanisms in dynamic thermocapillary liquid layers," J. Fluid Mech. 10, 3182-3186 (1986). 